\title{
Treatment patterns in Medicaid patients with schizophrenia initiated on a first- or second- generation long-acting injectable versus oral antipsychotic
}

\author{
This article was published in the following Dove Press journal: \\ Patient Preference and Adherence \\ 23 March 2017 \\ Number of times this article has been viewed
}

\section{Dominic Pilon' \\ Kruti Joshi \\ Neeta Tandon ${ }^{2}$ \\ Marie-Hélène Lafeuille \\ Rhiannon L Kamstra' \\ Bruno Emond' \\ Patrick Lefebvre ${ }^{2}$ \\ 'Groupe d'analyse, Ltée, Montréal, QC, Canada; ${ }^{2}$ Janssen Scientific Affairs, LLC, Titusville, NJ, USA}

\begin{abstract}
Background: Poor antipsychotic (AP) adherence is a key issue in patients with schizophrenia. First-generation antipsychotic (FGA) and second-generation antipsychotic (SGA) long-acting injectable therapies (LAI) may improve adherence compared to oral antipsychotics (OAP). The objective of the study was to compare treatment adherence and persistence in Medicaid patients with schizophrenia initiated on first-generation long-acting injectable therapies (FGA-LAI) or second-generation long-acting injectable therapies (SGA-LAI) versus OAP.
\end{abstract}

Methods: Adults with schizophrenia initiated on FGA-LAI, SGA-LAI, or OAP on or after January 2010 were identified using a six-state Medicaid database (January 2009-March 2015). Outcomes were assessed during the 12 months following treatment initiation. Index medication adherence was assessed using the proportion of days covered $\geq 80 \%$, while persistence was assessed as no gap of $\geq 30, \geq 60$, or $\geq 90$ days between days of supply. Outcomes were compared between FGA/ SGA-LAI and OAP cohorts using chi-squared tests and adjusted odds ratios (OR).

Results: During follow-up, AP polypharmacy was more common in FGA-LAI patients $(\mathrm{N}=1,089 ; 36 \% ; P=0.029)$ and less common in SGA-LAI patients $(\mathrm{N}=2,209 ; 27 \% ; P<0.001)$ versus OAP patients $(\mathrm{N}=20,478 ; 33 \%)$. After adjustment, SGA-LAI patients had $24 \%$ higher odds of adherence at 12 months (OR: $1.24 ; P<0.001$ ), in contrast to FGA-LAI patients who had $48 \%$ lower odds of adherence (OR: $0.52 ; P<0.001)$ relative to OAP patients. SGA-LAI patients were more likely to be persistent (no gap $\geq 60$ days) at 12 months than OAP patients (37\% vs 30\%; $P<0.001)$, but not FGA-LAI patients ( $31 \%$ vs $30 \% ; P=0.776)$. In comparison to OAP patients, SGA-LAI patients had $46 \%$ higher adjusted odds of persistence (no gap $\geq 60$ days; OR: 1.46 ; $P<0.001$ ), while FGA-LAI patients were not significantly different (OR: $0.95 ; P=0.501$ ).

Conclusion: Medicaid patients initiated on SGA-LAI demonstrated better treatment adherence and persistence compared to OAP patients, while those initiated on FGA-LAI did not show significant improvement in adherence or persistence and had more AP polypharmacy relative to OAP patients. These findings suggest the potential value of SGA-LAI in the treatment of schizophrenia.

Keywords: schizophrenia, long-acting injectable therapy, adherence, persistence, first generation, second generation

\section{Introduction}

According to the World Health Organization, schizophrenia is a severe mental disorder that is characterized by distortions in thinking, perception, emotions, language, sense of self, and behavior. ${ }^{1}$ While schizophrenia affects about $1.1 \%$ of the population in 
the United States, ${ }^{2}$ its burden in terms of health, social, and economic domains is important and affects not only patients but also family members, caregivers, and society ${ }^{3}$ largely due to the chronic, lifelong nature of the disease, and the frequent occurrence of relapses. ${ }^{4,5}$ Hence, in the United States, the overall cost of schizophrenia in 2013 was estimated as $\$ 155.7$ billion. $^{6}$

Antipsychotic (AP) medications are recommended as first-line treatment for schizophrenia and are classified as first-generation antipsychotics (FGA; or typical APs) and second-generation antipsychotics (SGA; or atypical APs). ${ }^{7}$ For each generation, both oral antipsychotics (OAP) and long-acting injectable therapies (LAI) are available. ${ }^{7}$ SGA have been proven effective to treat acute psychosis and are often preferred to FGA because they may be more effective at reducing the risk of future psychotic episodes ${ }^{8}$ and may be associated with fewer extrapyramidal side effects. ${ }^{7,9}$ In practice, both FGA and SGA are used in the management of schizophrenia. However, despite a wide range of available medications, poor AP adherence remains a key challenge in treating patients with schizophrenia and is a main driver of disease relapse. ${ }^{10,11}$ Given the importance of poor adherence and that it may, in part, explain relapses, there is a need to better understand factors that may influence adherence to AP treatment, including the choice of AP therapy.

LAI require less frequent administration than OAP (eg, once or twice monthly vs daily), illustrate effectiveness for the prescribed duration (eg, 1 month) without further action from the patient, and demonstrate compliance via direct monitoring by a health care practitioner. Although a recent meta-analysis of randomized clinical trials (RCTs) did not find that LAI reduced relapse compared to OAP in patients with schizophrenia, ${ }^{12}$ various authors have argued that RCTs may not be the most appropriate setting to assess medication adherence in patients with schizophrenia, since patients may be more likely to adhere to treatment in an RCT than in a real-world setting due to higher levels of monitoring and various incentives. ${ }^{12-15}$ Hence, in a meta-analysis of mirror-image studies (ie, studies comparing pre-post periods of OAP vs LAI use in the same patients), Kishimoto et al found a strong superiority of LAI compared to OAP in terms of preventing and decreasing the number of hospitalizations. ${ }^{15}$ Several observational studies have similarly demonstrated improved adherence and reduced re-hospitalizations associated with second-generation long-acting injectable therapies (SGA-LAI) versus OAP. ${ }^{13,16-18}$ In particular, a recent study of Medicaid patients by Marcus et al found that both firstgeneration long-acting injectable therapies (FGA-LAI) and SGA-LAI (in particular SGA-LAI) were associated with benefits in terms of adherence and re-hospitalizations among a population of recently hospitalized, non-adherent patients with schizophrenia. ${ }^{18}$

In the context of the current literature, there remains a need to better understand the real-world impact of FGA and SGA-LAI in a broader population of patients with schizophrenia and a need to account for recently approved SGA-LAI (ie, aripiprazole LAI). The present study compared treatment adherence and persistence, as well as treatment patterns, in a population of Medicaid patients diagnosed with schizophrenia between patients initiated on FGA-LAI or SGA-LAI and patients initiated on OAP.

\section{Methods \\ Data source}

This study was conducted using databases of medical and pharmacy claims of Medicaid beneficiaries in Florida, Iowa, Kansas, Mississippi, Missouri, and New Jersey. Data were available from September 1, 2008 through March 31, 2015 for all states except for Florida, which had available data until June 30, 2013, and New Jersey, which had available data until March 31, 2014. Available data included patient eligibility records (eg, age, sex, race, enrollment start end/ dates), medical claims (eg, type of service, date of service, International Classification of Diseases, Ninth Revision, Clinical Modification [ICD-9-CM] diagnoses, Current Procedural Terminology procedure codes, and Healthcare Common Procedure Coding System [HCPCS] codes), and prescription drug claims (eg, days of medication supplied, date of prescription fills, and National Drug Codes [NDC]). All data were de-identified and in compliance with the Health Insurance Portability and Accountability Act.

\section{Study design and patient selection}

A retrospective longitudinal cohort study design was used to compare treatment patterns, adherence, and persistence in adult Medicaid beneficiaries with schizophrenia who initiated an FGA-LAI versus an OAP, and those who initiated an SGA-LAI versus an OAP. The FGA-LAI cohort encompassed fluphenazine decanoate and haloperidol decanoate, while the SGA-LAI cohort encompassed aripiprazole, olanzapine, paliperidone palmitate, and risperidone. Specific LAI agents were identified using NDC codes for prescription claims and HCPCS codes for medical claims. OAP medications included both FGA and SGA and were identified as medications with a generic product identifier beginning with " 59 " and with an oral route of administration. To be included in the study, patients were required to meet the following criteria: 1) have at least two pharmacy/medical 
claims for the same LAI or at least two pharmacy claims for the same OAP agent within 90 days starting from January 1,2010 (the date of the first claim was defined as the index date) with no claims for the same agent in the previous 12 months, 2) have at least two diagnoses for schizophrenia (ICD-9-CM codes: 295.xx) during their continuous Medicaid enrollment, 3 ) be at least 18 years of age on the index date, 4) have at least 12 months of continuous Medicaid enrollment prior to the index date (baseline period; ie, pre-index date), and 5) have at least 12 months of post-index continuous Medicaid enrollment. Continuous enrollment for each patient was identified prior to sample selection and was defined as the longest period of continuous enrollment during the study period (2009-2015).

Treatment cohorts (FGA-LAI, SGA-LAI, or OAP) were defined by the agent initiated on the index date. As per the definition for treatment initiation, patients had no baseline use of their index AP. However, patients were permitted to have other baseline AP use, most accurately reflecting the real-world patient population. Each patient contributed only once to the analysis. Outcomes were evaluated during a fixed 12-month observation period including and following the index date. Baseline demographics and clinical characteristics were evaluated during the baseline period.

A sensitivity analysis only comparing each LAI cohort with its respective generation of OAP (ie, FGA-LAI vs FGAOAP and SGA-LAI vs SGA-OAP) was also conducted.

\section{Study outcomes}

Outcome measures included index medication adherence and persistence, as well as treatment patterns compared between FGA-LAI and OAP patients, and between SGA-LAI and OAP patients.

Treatment patterns evaluated during the observation period included the number of AP prescription fills (for all APs and for the index AP only), psychiatric medication use (other than the index agent), the presence of AP polypharmacy (ie, overlapping coverage of $\geq 2$ unique AP agents for $\geq 60$ consecutive days with no more than a 7-day gap), and the presence of psychiatric polypharmacy (ie, overlapping coverage of $\geq 1$ AP agent and $\geq 1$ anxiolytic, antidepressant, or mood stabilizer for $\geq 60$ consecutive days with no more than a 7-day gap). The criterion of at least 60 days of medication overlap was used to exclude short-term polypharmacy related to medication switching. The number of mental health diagnoses at baseline was defined as the number of unique 4-digit ICD-9-CM diagnoses (including diagnoses related to schizophrenia, psychosis, and substance use).
Adherence and persistence were evaluated at 12 months post-index date for the index agent. Adherent patients were defined as patients with a proportion of days covered $(\mathrm{PDC}) \geq 80 \%$. PDC was defined as the number of non-overlapping days of supply divided by the number of days in the observation period (365 days). Persistence on the index AP agent at 12 months was measured by assessing the proportion of patients with no continuous gap of at least 30, 60, or 90 days between days of supply of the index medication.

\section{Statistical analysis}

Pairwise comparisons of FGA-LAI versus OAP patients and SGA-LAI versus OAP patients were conducted using both unadjusted (descriptive) and adjusted analyses.

\section{Descriptive analysis}

Baseline characteristics, treatment patterns, and unadjusted adherence and persistence outcomes were reported using mean, median, and standard deviation for continuous variables and frequency and proportion for categorical variables, and compared using Wilcoxon rank sum tests or chi-squared tests, respectively.

\section{Adjusted analysis}

The probability of adherence (ie, $\mathrm{PDC} \geq 80 \%$ ) and persistence was estimated using multivariable logistic regression models adjusting for the following baseline variables: age, sex, race, state, region, year of index date, presence of dual insurance eligibility, Quan-Charlson comorbidity index (Quan-CCI), type of schizophrenia diagnosis, specific comorbidities, AP adherence (ie, $\mathrm{PDC} \geq 80 \%$ ), number of unique mental health diagnoses, number of AP agents, use of AP and concomitant medication, presence of AP polypharmacy, total pharmacy costs, total medical costs, the number of mental health institute visits, and the number of 1-day mental health institute visits.

All analyses were performed using SAS software, Version 9.3 of the SAS System for Windows, SAS Institute Inc., Cary, NC, USA.

\section{Ethics approval}

As this was an analysis of claims data, institutional review board (IRB) approval was not required. Per Title 45 of CFR, Part 46.101(b)(4), the administrative claims data analysis of our study is exempt from the IRB review for two reasons: 1) it is a retrospective analysis of existing data (hence no patient intervention or interaction) and 2) no patient-identifiable information is included in the claims dataset (available from the Office for Human Research Protections: https://www.hhs.gov/ ohrp/regulations-and-policy/regulations/45-cfr-46/\#46.101). 


\begin{tabular}{|c|c|c|c|c|c|}
\hline \multicolumn{6}{|c|}{ Patients with $\geq 2$ schizophrenia diagnoses (ICD-9-CM code: 295.xx) on or after January 1, 2009} \\
\hline \multicolumn{6}{|c|}{$\mathrm{N}=152,977$} \\
\hline \multicolumn{6}{|c|}{ Patients with $\geq 1$ LAl $^{\text {a }}$ or OAP claim on or after January 1, 2009} \\
\hline \multicolumn{6}{|c|}{$\mathrm{N}=95,818(62.6 \%)$} \\
\hline \multicolumn{6}{|c|}{$\begin{array}{c}\text { Patients initiated }{ }^{b} \text { on either an LAI or an OAP on or after January 1, } 2010 \text { with the first claim } \\
\text { being the index date }\end{array}$} \\
\hline \multicolumn{6}{|c|}{$\mathrm{N}=57,058(59.5 \%)$} \\
\hline \multicolumn{2}{|c|}{ Patients initiating an LAI } & \multicolumn{4}{|c|}{ Patients initiating an OAP } \\
\hline \multicolumn{2}{|c|}{$\mathrm{N}=5,950(10.4 \%)$} & \multicolumn{4}{|c|}{$\mathrm{N}=51,108(89.6 \%)$} \\
\hline \multicolumn{2}{|c|}{$\begin{array}{l}\geq 1 \text { schizophrenia diagnosis (ICD-9-CM code: } \\
295 . x x) \text { within } 12 \text { months before the index date }\end{array}$} & \multicolumn{4}{|c|}{$\begin{array}{l}\geq 1 \text { schizophrenia diagnosis (ICD-9-CM code: } \\
295 . x x \text { ) within } 12 \text { months before the index date }\end{array}$} \\
\hline \multicolumn{2}{|c|}{$\mathrm{N}=4,927(82.8 \%)$} & \multicolumn{4}{|c|}{$\mathrm{N}=34,161(66.8 \%)$} \\
\hline \multicolumn{2}{|c|}{$\geq 18$ years of age on the index date } & \multicolumn{4}{|c|}{$\geq 18$ years of age on the index date } \\
\hline \multicolumn{2}{|c|}{$\mathrm{N}=4,897(99.4 \%)$} & \multicolumn{4}{|c|}{$\mathrm{N}=33,318(97.5 \%)$} \\
\hline \multicolumn{2}{|c|}{$\begin{array}{c}\text { Continuous eligibility for } \geq 12 \text { months prior to } \\
\text { the index date (baseline period) }\end{array}$} & \multicolumn{4}{|c|}{$\begin{array}{c}\text { Continuous eligibility for } \geq 12 \text { months prior to } \\
\text { the index date (baseline period) }\end{array}$} \\
\hline \multicolumn{2}{|c|}{$\mathrm{N}=3,788(77.4 \%)$} & \multicolumn{4}{|c|}{$\mathrm{N}=24,006(72.1 \%)$} \\
\hline \multicolumn{2}{|c|}{$\begin{array}{l}\text { Continuous eligibility for } \geq 12 \text { months after the } \\
\text { index date (observation period) }\end{array}$} & \multicolumn{4}{|c|}{$\begin{array}{l}\text { Continuous eligibility for } \geq 12 \text { months after the } \\
\text { index date (observation period) }\end{array}$} \\
\hline \multicolumn{2}{|c|}{$\mathrm{N}=3,298(87.1 \%)$} & \multicolumn{4}{|c|}{$\mathrm{N}=20,478(85.3 \%)$} \\
\hline $\begin{array}{l}\text { First-generation } \\
\text { LAI (FGA-LAI) }\end{array}$ & $\begin{array}{l}\text { Second-generation } \\
\text { LAI (SGA-LAI) }\end{array}$ & $\begin{array}{l}\text { First-gen } \\
\text { OAP (FG) }\end{array}$ & $\begin{array}{l}\text { eration } \\
\text { A-OAP) }\end{array}$ & $\begin{array}{l}\text { Second-ge } \\
\text { OAP (SG }\end{array}$ & $\begin{array}{l}\text { eneration } \\
\text { SA-OAP) }\end{array}$ \\
\hline $\mathrm{N}=1,089(33.0 \%)$ & $\mathrm{N}=2,209(67.0 \%)$ & $\mathrm{N}=4,357$ & $21.3 \%)$ & \multicolumn{2}{|c|}{$\mathrm{N}=16,121(78.7 \%)$} \\
\hline Specific agent, $\mathbf{N}(\%)$ & Specific agent, $\mathbf{N}(\%)$ & \multicolumn{2}{|c|}{$\begin{array}{r}\mathrm{N}=4,357(21.3 \%) \\
\text { Specific agent, } \mathrm{N}(\%)\end{array}$} & Specific ager & nt, $\mathbf{N}(\%)$ \\
\hline Haloperidol $766(70.3)$ & Aripiprazole & Chlorpromazine & $525(12.0)$ & Aripiprazole & $2,495(15.5)$ \\
\hline Fluphenazine 323 (29.7) & Olanzapine $\quad 26(1.2)$ & Fluphenazine & $682(15.7)$ & Asenapine & $626(3.9)$ \\
\hline & Paliperidone 1,450 (65.6) & Haloperidol & $2,210(50.7)$ & Clozapine & $529(3.3)$ \\
\hline & Risperidone $712(32.2)$ & Loxapine & $202(4.6)$ & Iloperidone & $389(2.4)$ \\
\hline & & Molindone & $2(0.0)$ & Lurasidone & $645(4.0)$ \\
\hline & & Perphenazine & $383(8.8)$ & Olanzapine & $2,113(13.1)$ \\
\hline & & Thioridazine & $64(1.5)$ & Paliperidone & $1,076(6.7)$ \\
\hline & & Thiothixene & $159(3.6)$ & Quetiapine & $3,006(18.6)$ \\
\hline & & Trifluoperazine & $130(3.0)$ & Risperidone & $3,939(24.4)$ \\
\hline & & & & Ziprasidone & $1,303(8.1)$ \\
\hline
\end{tabular}

Figure I Identification of the study population.

Notes: ${ }^{2} \mathrm{LAl}$ agents include first-generation: fluphenazine LAI, haloperidol LAl; second-generation: olanzapine LAI, risperidone LAI, paliperidone LAI, and aripiprazole LAI. bInitiation of an antipsychotic agent is defined as having $\geq 2$ claims for the same agent within 90 days and no claim for the same agent during the 12 months before the first claim. Abbreviations: FGA, first-generation antipsychotics; ICD-9-CM, International Classification of Diseases, Ninth Revision, Clinical Modification; LAI, long-acting injectable therapies; OAP, oral antipsychotics; SGA, second-generation antipsychotics. 
Moreover, the requirement for patient consent was not applicable given the retrospective nature of the study and the use of deidentified data.

\section{Results}

\section{Demographic and clinical characteristics}

A total of 23,776 patients with schizophrenia met all inclusion criteria. Of them, 20,478 (86.1\%) were initiated on OAP, 1,089 (4.6\%) were initiated on FGA-LAI, and 2,209 (9.3\%) were initiated on SGA-LAI. Two-thirds of patients initiating an LAI were initiated on an SGA-LAI (Figure 1).

A large majority of patients initiated on OAP received a second-generation OAP (78.7\%), while the most commonly prescribed agent among patients initiated on FGA-LAI was haloperidol decanoate $(70.3 \%)$, and the most commonly prescribed agent among patients initiated on SGA-LAI was paliperidone palmitate (65.6\%; Figure 1). The most common first- and second-generation OAP agents initiated were haloperidol (50.7\%) and risperidone $(24.4 \%)$, respectively (Figure 1).

On an average, patients initiated on SGA-LAI were younger than those initiated on OAP (42.2 vs 44.8 years; $P<0.001$ ), while mean age appeared similar between FGALAI and OAP patients (Table 1). A higher proportion of patients initiated on OAP were females as compared to FGA-LAI patients (48.9\% vs $39.7 \% ; P<0.001$ ) or SGA-LAI patients (48.9\% vs 39.6\%; $P<0.001$; Table 1$)$.

On an average, patients initiated on OAP had a higher baseline (ie, pre-index date) comorbidity burden compared to FGA or SGA-LAI patients, with a higher mean Quan-CCI (1.3 vs 0.9 [FGA-LAI] or 0.8 [SGA-LAI]; all $P<0.001$ ), a higher mean number of unique mental health diagnoses (9.3 vs 8.0 [FGA-LAI] or 8.1 [SGA-LAI]; all $P<0.001$ ), and a higher proportion of patients with non-AP psychiatric medication use (ie, anxiolytics, antidepressants, or mood stabilizers; $76.9 \%$ vs $65.2 \%$ [FGA-LAI] or $68.4 \%$ [SGA-LAI]; all $P<0.001$; Table 1).

However, during baseline, patients initiated on SGA-LAI were more likely to have used an AP agent (79.8\% vs 68.0\%; $P<0.001$ ), to have received more unique AP agents (mean: 1.5 vs $1.1 ; P<0.001)$, and were more likely to have AP polypharmacy $(19.5 \%$ vs $14.0 \% ; P<0.001)$, compared to the OAP cohort (Table 1). Lastly, during baseline and among patients with pre-index AP use, a higher proportion of the OAP cohort was adherent (PDC $\geq 80 \%$ ) as compared to the SGA-LAI cohort (37.5\% vs 33.0\% [SGA-LAI]; $P<0.001$ ), while baseline adherence in the FGA-LAI cohort was not significantly different (37.5 vs $34.4 \%$ [FGA-LAI]; $P=0.106$; Table 1 ).

\section{Treatment patterns}

Table 2 describes the treatment patterns observed in the three cohorts during the 12-month observation period. Following index treatment initiation, SGA-LAI patients had significantly more claims for the index AP as compared to OAP patients (9.1 vs 8.3 claims; $P<0.001$ ) and were less likely to use an AP other than the index medication (63.1\% vs $67.5 \%$; $P<0.001$; Table 2). Furthermore, both FGA-LAI and SGA-LAI patients were less likely to use adjunctive therapy (ie, anxiolytics, antidepressants, or mood stabilizers) during follow-up (69.5\% and $68.8 \%$, respectively) as compared to OAP patients (89.8\%; all $P<0.001$; Table 2). Finally, relative to the OAP cohort, AP polypharmacy was less common in the SGA-LAI cohort $(26.5 \%$ vs $32.8 \% ; P<0.001)$ and more common in the FGA-LAI cohort (36.0\% vs 32.8\%; $P=0.029$; Table 2$)$.

\section{Adherence and persistence}

Figures 2 and 3 illustrate the observed (unadjusted) results of adherence and persistence to index treatment during the 12-month follow-up period.

Before adjustment, a higher proportion of SGA-LAI patients were adherent to index medication at 12 months (PDC $\geq 80 \%$ : $27.2 \%$ vs $24.6 \% ; P=0.008$ ), while a lower proportion of FGALAI patients were adherent at 12 months (PDC $\geq 80 \%$ : $15.8 \%$ vs $24.6 \% ; P<0.001$ ) compared to OAP patients (Figure 2). On an average, adherence to the index medication was higher for SGA-LAI patients (mean PDC: $52.1 \%$ vs $49.8 \%$; $P=0.007$ ) but similar for FGA-LAI patients (mean PDC: $48.2 \%$ vs $49.8 \%$; $P=0.160$ ) as compared to OAP patients (Figure 2).

Similarly, persistence to index medication at 12 months measured by no gap of at least 30 days was higher for SGA-LAI patients (no gap $\geq 30: 23.7 \%$ vs $20.6 \% ; P<0.001$ ), but lower for FGA-LAI patients (no gap $\geq 30$ : $13.6 \%$ vs $20.6 \% ; P<0.001$ ) compared to OAP patients (Figure 3 ). Persistence using no gaps of at least 60 days and 90 days were similarly higher for SGA-LAI patients compared to OAP patients (no gap $\geq 60$ : $37.1 \%$ vs $30.2 \%$; $P<0.001$ and no gap $\geq 90$ : $42.9 \%$ vs $36.9 \%$; $P<0.001$; Figure 3 ). Using gaps of at least 90 days, persistence was higher for FGA-LAI patients compared to OAP patients $(41.2 \%$ vs $36.9 \% ; P<0.004$; Figure 3 ).

Given the differences in baseline characteristics observed between cohorts (Table 1), multivariable regression was used to generate adjusted comparisons between SGA and FGA-LAI patients versus OAP patients. Figure 4 shows adjusted results on adherence and persistence to index treatment during the 12-month follow-up period. After adjustment, patients initiated on SGA-LAI had 24\% higher 
Table I Demographic and clinical characteristics evaluated during the I2-month baseline period

\begin{tabular}{|c|c|c|c|c|c|}
\hline \multirow[t]{2}{*}{ Characteristic } & \multirow{2}{*}{$\frac{\operatorname{OAP}(\mathrm{A})}{(\mathrm{N}=20,478)}$} & \multicolumn{2}{|l|}{ FGA-LAI (B) } & \multicolumn{2}{|l|}{ SGA-LAI (C) } \\
\hline & & $(\mathrm{N}=1,089)$ & $\begin{array}{l}P \text {-value } \\
\text { (A) vs (B) }\end{array}$ & $(\mathrm{N}=2,209)$ & $\begin{array}{l}P_{\text {-value }}{ }^{\text {a }} \\
\text { (A) vs (C) }\end{array}$ \\
\hline Age on index date (years), mean \pm SD (median) & $44.8 \pm 13.2(46.5)$ & $45.5 \pm 12.5(47.0)$ & 0.080 & $42.2 \pm 12.8(42.7)$ & $<0.00 I^{*}$ \\
\hline Female, $\mathrm{n}(\%)$ & $10,006(48.9)$ & $432(39.7)$ & $<0.00 I^{*}$ & $875(39.6)$ & $<0.001 *$ \\
\hline \multicolumn{6}{|l|}{ Race, n (\%) } \\
\hline White & $10,767(52.6)$ & $417(38.3)$ & $<0.00 I^{*}$ & $\mathrm{I}, 06 \mathrm{I}(48.0)$ & $<0.001 *$ \\
\hline Black & $6,913(33.8)$ & $524(48.1)$ & $<0.001 *$ & $900(40.7)$ & $<0.001 *$ \\
\hline Hispanic & $867(4.2)$ & $44(4.0)$ & 0.757 & $21(1.0)$ & $<0.001 *$ \\
\hline Other & $\mathrm{I}, 055(5.2)$ & $38(3.5)$ & $0.015^{*}$ & $156(7.1)$ & $<0.001 *$ \\
\hline Unknown & $876(4.3)$ & $66(6.1)$ & $0.005^{*}$ & $7 \mid(3.2)$ & $0.018^{*}$ \\
\hline \multicolumn{6}{|l|}{ State, n (\%) } \\
\hline Florida & $3,543(17.3)$ & $217(19.9)$ & $0.026^{*}$ & $93(4.2)$ & $<0.00 I^{*}$ \\
\hline lowa & $907(4.4)$ & $128(\mid 1.8)$ & $<0.00 \mathrm{I} *$ & $172(7.8)$ & $<0.00 \mathrm{I} *$ \\
\hline Kansas & $1,392(6.8)$ & $42(3.9)$ & $0.00 I^{*}$ & $202(9.1)$ & $<0.001 *$ \\
\hline Mississippi & I,895 (9.3) & $152(14.0)$ & $<0.00 \mathrm{I} *$ & $253(I I .5)$ & $<0.001 *$ \\
\hline Missouri & $8,445(4 \mid .2)$ & $376(34.5)$ & $<0.00$ I* & $980(44.4)$ & $0.005^{*}$ \\
\hline New Jersey & $4,296(21.0)$ & $174(16.0)$ & $<0.00 I^{*}$ & $509(23.0)$ & $0.024 *$ \\
\hline \multicolumn{6}{|l|}{ Region characteristics, n (\%) } \\
\hline Urban & $12,019(58.7)$ & $639(58.7)$ & 0.992 & I,247 (56.5) & $0.042 *$ \\
\hline Suburban & $5,425(26.5)$ & $289(26.5)$ & 0.973 & $572(25.9)$ & 0.545 \\
\hline Rural & $3,034(14.8)$ & $161(14.8)$ & 0.977 & $390(17.7)$ & $<0.00$ I* \\
\hline \multicolumn{6}{|l|}{ Insurance eligibility, n (\%) } \\
\hline Capitated or dual coverage & $12,693(62.0)$ & $632(58.0)$ & $0.009 *$ & I,466 (66.4) & $<0.00$ I* \\
\hline Capitated & $7,750(37.8)$ & $338(31.0)$ & $<0.00 I^{*}$ & $864(39.1)$ & 0.244 \\
\hline Dual coverage & $7,675(37.5)$ & $423(38.8)$ & 0.365 & $910(41.2)$ & $<0.00 \mathrm{I} *$ \\
\hline \multicolumn{6}{|l|}{ Year of index date, n (\%) } \\
\hline 2010 & $9,726(47.5)$ & $460(42.2)$ & $<0.001 *$ & I,080 (48.9) & 0.212 \\
\hline 2011 & $5,79 \mid(28.3)$ & $286(26.3)$ & 0.149 & $517(23.4)$ & $<0.001 *$ \\
\hline 2012 & $2,976(14.5)$ & $209(19.2)$ & $<0.001 *$ & $330(14.9)$ & 0.607 \\
\hline 2013 & $\mathrm{I}, 575(7.7)$ & $101(9.3)$ & 0.057 & $203(9.2)$ & $0.013 *$ \\
\hline 2014 & $410(2.0)$ & $33(3.0)$ & $0.020^{*}$ & $79(3.6)$ & $<0.001 *$ \\
\hline Quan-CCl, mean \pm SD (median) & $1.3 \pm 1.8(1.0)$ & $0.9 \pm 1.5(0.0)$ & $<0.001 *$ & $0.8 \pm 1.3(0.0)$ & $<0.001 *$ \\
\hline Number of unique mental health diagnoses, ${ }^{\mathrm{b}}$ mean \pm SD (median) & $9.3 \pm 8.6(7.0)$ & $8.0 \pm 7.8(6.0)$ & $<0.001 *$ & $8.1 \pm 7.6(6.0)$ & $<0.001 *$ \\
\hline Number of unique $\mathrm{AP}$ agents received, mean $\pm \mathrm{SD}$ (median) & $I . I \pm I . I(I .0)$ & $1.3 \pm 1.3(1.0)$ & 0.217 & $1.5 \pm 1.2(1.0)$ & $<0.001 *$ \\
\hline AP use, $n(\%)$ & $13,927(68.0)$ & $697(64.0)$ & $0.006 *$ & ।,763 (79.8) & $<0.001 *$ \\
\hline FGA oral and short-term injectable APs & $4,096(20.0)$ & $4 \mid 3(37.9)$ & $<0.00 I^{*}$ & $393(17.8)$ & $0.013^{*}$ \\
\hline SGA oral and short-term injectable APs & $11,906(58.1)$ & $514(47.2)$ & $<0.00$ I* & $\mathrm{I}, 447(65.5)$ & $<0.00$ I* \\
\hline FGA-LAI & $\mathrm{I}, 578(7.7)$ & $74(6.8)$ & 0.271 & $291(13.2)$ & $<0.001 *$ \\
\hline SGA-LAI & I, II $5(5.4)$ & $73(6.7)$ & 0.076 & $488(22.1)$ & $<0.001 *$ \\
\hline \multicolumn{6}{|l|}{ Proportion of days covered (PDC) by any AP agent, $n(\%)$} \\
\hline $\mathrm{PDC}<80 \%$ & $8,709(62.5)$ & $457(65.6)$ & 0.106 & I, I82 (67.0) & $<0.001 *$ \\
\hline $\mathrm{PDC} \geq 80 \%^{\mathrm{c}}$ & $5,218(37.5)$ & $240(34.4)$ & 0.106 & $581(33.0)$ & $<0.001 *$ \\
\hline AP polypharmacy present, ${ }^{d}$ n (\%) & $2,867(14.0)$ & $20 \mid(\mid 8.5)$ & $<0.00 I^{*}$ & $430(19.5)$ & $<0.001 *$ \\
\hline Other psychiatric medication use, $\mathrm{n}(\%)$ & I5,746 (76.9) & $710(65.2)$ & $<0.001 *$ & $\mathrm{I}, 5 \mathrm{I} 2(68.4)$ & $<0.001 *$ \\
\hline Antidepressants & II,486 (56.I) & $430(39.5)$ & $<0.001 *$ & $\mathrm{I}, 032(46.7)$ & $<0.001 *$ \\
\hline Anxiolytics & $10,560(51.6)$ & $429(39.4)$ & $<0.001 *$ & $849(38.4)$ & $<0.001 *$ \\
\hline Mood stabilizers & $8,504(4 \mid .5)$ & $398(36.5)$ & $0.001 *$ & $826(37.4)$ & $<0.001 *$ \\
\hline Number of unique psychiatric agents received, ${ }^{\mathrm{e}}$ mean $\pm \mathrm{SD}$ (median) & $3.4 \pm 2.7(3.0)$ & $2.9 \pm 2.8(2.0)$ & $<0.001 *$ & $3.2 \pm 2.5(3.0)$ & $0.001 *$ \\
\hline Psychiatric polypharmacy present, ${ }^{f} n(\%)$ & $8,932(43.6)$ & $383(35.2)$ & $<0.001 *$ & $884(40.0)$ & $0.001 *$ \\
\hline
\end{tabular}

Notes: ${ }^{* P}$-value $<0.05$ (versus OAP). aP-value refers to a comparison with the OAP reference group, calculated using the Pearson chi-squared test (categorical variables) and Wilcoxon rank-sum test (continuous variables). ${ }^{\circ}$ The number of unique 4-digit ICD-9-CM diagnoses observed (including schizophrenia, psychosis, and substance use-related diagnoses). 'The denominator for the proportion of patients with a PDC $<80 \%$ or a PDC $\geq 80 \%$ is the number of patients with a PDC $>0$. ${ }^{\mathrm{d}} \mathrm{AP}$ polypharmacy is defined as having overlapping coverage of $\geq 2$ unique AP agents for at least 60 consecutive days with no gaps larger than 7 days. elncludes mood stabilizers, anxiolytics, antidepressants, and AP. 'Psychiatric polypharmacy is defined as having overlapping coverage of $\geq 1 \mathrm{AP}$ agent and $\geq 1$ anxiolytic, antidepressant, or mood stabilizer for at least 60 consecutive days with no gaps larger than 7 days.

Abbreviations: AP, antipsychotics; Quan-CCl, Quan-Charlson comorbidity index; FGA, first-generation antipsychotics; ICD-9-CM, International Classification of Diseases, Ninth Revision, Clinical Modification; LAI, long-acting injectable therapies; OAP, oral antipsychotics; PDC, proportion of days covered; SD, standard deviation; SGA, secondgeneration antipsychotics. 
Table 2 Treatment patterns observed during the 12-month follow-up period

\begin{tabular}{|c|c|c|c|c|c|}
\hline \multirow[t]{2}{*}{ Characteristic } & \multirow{2}{*}{$\frac{\operatorname{OAP}(\mathrm{A})}{(\mathrm{N}=20,478)}$} & \multicolumn{2}{|l|}{ FGA-LAI (B) } & \multicolumn{2}{|l|}{ SGA-LAI (C) } \\
\hline & & $(\mathrm{N}=1,089)$ & $\begin{array}{l}P \text {-value }{ }^{a} \\
\text { (A) vs (B) }\end{array}$ & $(\mathrm{N}=2,209)$ & $\begin{array}{l}P \text {-value }{ }^{a} \\
\text { (A) vs (C) }\end{array}$ \\
\hline Number of AP prescription fills, mean \pm SD (median) & $16.2 \pm 13.6(13.0)$ & $16.8 \pm 13.6(13.0)$ & $0.018^{*}$ & $|5.1 \pm| 1.3(13.0)$ & 0.170 \\
\hline Number of prescription fills for the index drug, mean \pm SD (median) & $8.3 \pm 7.1(7.0)$ & $7.7 \pm 5.0(7.0)$ & 0.293 & $9.1 \pm 5.9(8.0)$ & $<0.001 *$ \\
\hline Number of unique $\mathrm{AP}$ agents received, mean $\pm \mathrm{SD}$ (median) & $2.2 \pm 1.2(2.0)$ & $2.2 \pm 1.2(2.0)$ & 0.261 & $2.1 \pm 1.2(2.0)$ & $<0.00 I^{*}$ \\
\hline PDC for index medication (\%), mean \pm SD & $49.8 \pm 30.2(44.1)$ & $48.2 \pm 27.1(45.2)$ & 0.160 & $52.1 \pm 29.7(49.3)$ & $0.007^{*}$ \\
\hline Psychiatric medication use during follow-up (excluding index drug), n (\%) & $19,449(95.0)$ & $883(81.1)$ & $<0.00 I^{*}$ & I,77I (80.2) & $<0.00 I^{*}$ \\
\hline AP use & $13,832(67.5)$ & $733(67.3)$ & 0.871 & I,394 (63.1) & $<0.00 I^{*}$ \\
\hline FGA oral and short-term injectable APs & $4,44 \mid(2 \mid .7)$ & $45 I(4 I .4)$ & $<0.001 *$ & $393(17.8)$ & $<0.001 *$ \\
\hline SGA oral and short-term injectable APs & II,037 (53.9) & $515(47.3)$ & $<0.001 *$ & $1,207(54.6)$ & 0.505 \\
\hline FGA-LAI & $1,924(9.4)$ & $59(5.4)$ & $<0.001 *$ & $115(5.2)$ & $<0.001 *$ \\
\hline SGA-LAI & $1,870(9.1)$ & $69(6.3)$ & $0.002^{*}$ & $143(6.5)$ & $<0.00 I^{*}$ \\
\hline Adjunctive therapy use & |8,39| (89.8) & $757(69.5)$ & $<0.001 *$ & $1,519(68.8)$ & $<0.00 I^{*}$ \\
\hline Anxiolytics & $12,262(59.9)$ & $473(43.4)$ & $<0.001 *$ & $851(38.5)$ & $<0.00 I^{*}$ \\
\hline Mood stabilizers & $10,754(52.5)$ & $419(38.5)$ & $<0.001 *$ & $830(37.6)$ & $<0.00 I^{*}$ \\
\hline Antidepressants & |4, I $37(69.0)$ & $475(43.6)$ & $<0.001 *$ & $1,056(47.8)$ & $<0.00 I^{*}$ \\
\hline AP polypharmacy present, ${ }^{b} \mathrm{n}(\%)$ & $6,717(32.8)$ & $392(36.0)$ & $0.029 *$ & $585(26.5)$ & $<0.001 *$ \\
\hline Psychiatric polypharmacy present, ${ }^{c}$ n (\%) & $14,373(70.2)$ & $546(50.1)$ & $<0.001 *$ & $\mathrm{I}, 033(46.8)$ & $<0.00 I^{*}$ \\
\hline
\end{tabular}

Notes: $* P$-value $<0.05$ (versus OAP). aP-value refers to a comparison with the OAP reference group, calculated using the Pearson chi-squared test (categorical variables) and Wilcoxon rank-sum test (continuous variables). ${ }^{b} A P$ polypharmacy is defined as having overlapping coverage of $\geq 2$ unique AP agents for at least 60 consecutive days with no gaps larger than 7 days. 'Psychiatric polypharmacy is defined as having overlapping coverage of $\geq I$ AP agent and $\geq I$ anxiolytic, antidepressant, or mood stabilizer for at least 60 consecutive days with no gaps larger than 7 days.

Abbreviations: AP, antipsychotics; FGA, first-generation antipsychotics; LAI, long-acting injectable therapies; OAP, oral antipsychotics; PDC, proportion of days covered; SGA, second-generation antipsychotics; SD, standard deviation.

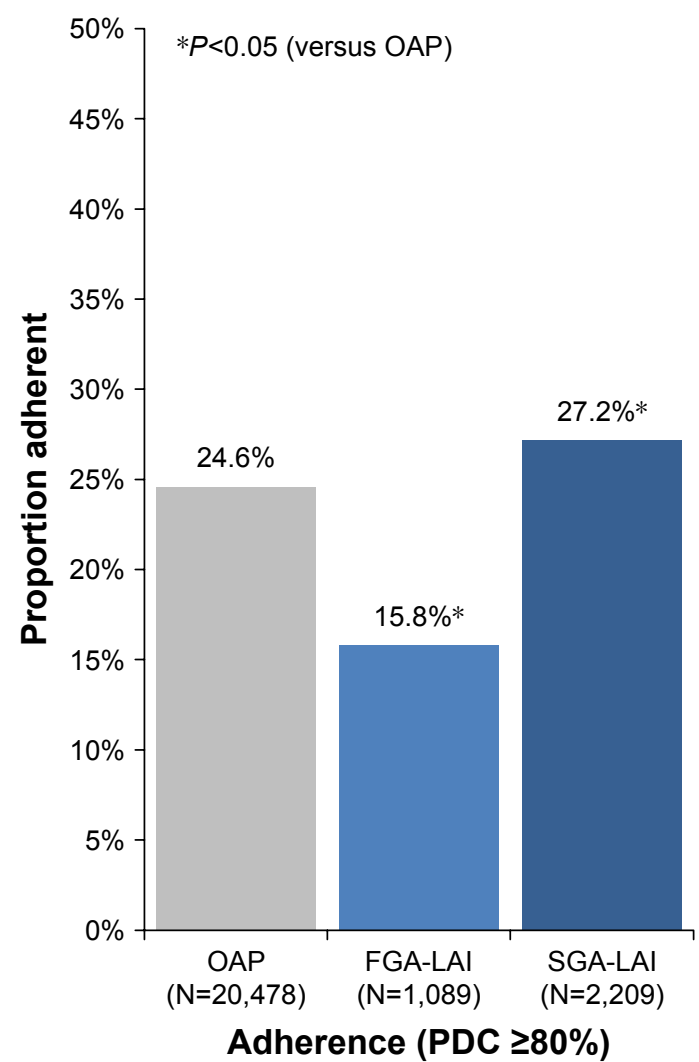

Figure 2 Observed adherence (PDC $\geq 80 \%$ ) on index treatment during the 12 -month follow-up period.

Abbreviations: FGA-LAI, first-generation long-acting injectable therapies; OAP, oral antipsychotics; PDC, proportion of days covered; SGA-LAI, second-generation long-acting injectable therapies. odds of being adherent at 12 months (odds ratio [OR]: 1.24; $P<0.001)$, whereas FGA-LAI patients had $48 \%$ lower odds of adherence (OR: $0.52 ; P<0.001$ ) relative to OAP patients (Figure 4). Similarly, SGA-LAI patients had $31 \%$ higher odds of persistence (no gap $\geq 30$ days) compared to OAP patients (OR: $1.31 ; P<0.001$ ), while FGA-LAI patients had $45 \%$ lower odds of persistence (no gap $\geq 30$ days) compared to OAP patients (OR: $0.55 ; P=0.501$; Figure 4 ). Using definitions of no gap $\geq 60$ days or $\geq 90$ days, patients initiated on SGA-LAI had consistently higher odds of persistence (odds of persistence $46 \%$ and $35 \%$ greater, respectively) compared to patients initiated on OAP (OR for no gap $\geq 60$ days: $1.46, P<0.001$; OR for no gap $\geq 90$ days: $1.35, P<0.001$; Figure 4). As for patients initiated on FGA-LAI, they had similar odds of being persistent as measured with no gap $\geq 60$ or $\geq 90$ days (OR for no gap $\geq 60$ days: $0.95 ; P=0.501$; OR for no gap $\geq 90$ days: $1.13, P=0.071$ ) as compared to patients initiated on OAP (Figure 4).

Similar results were found when comparing adherence and persistence outcomes in each LAI cohort with its respective generation of OAP (ie, FGA-LAI vs FGA-OAP and SGA-LAI vs SGA-OAP).

\section{Discussion}

This retrospective study of nearly 24,000 Medicaid patients diagnosed with schizophrenia initiated on AP medication in 


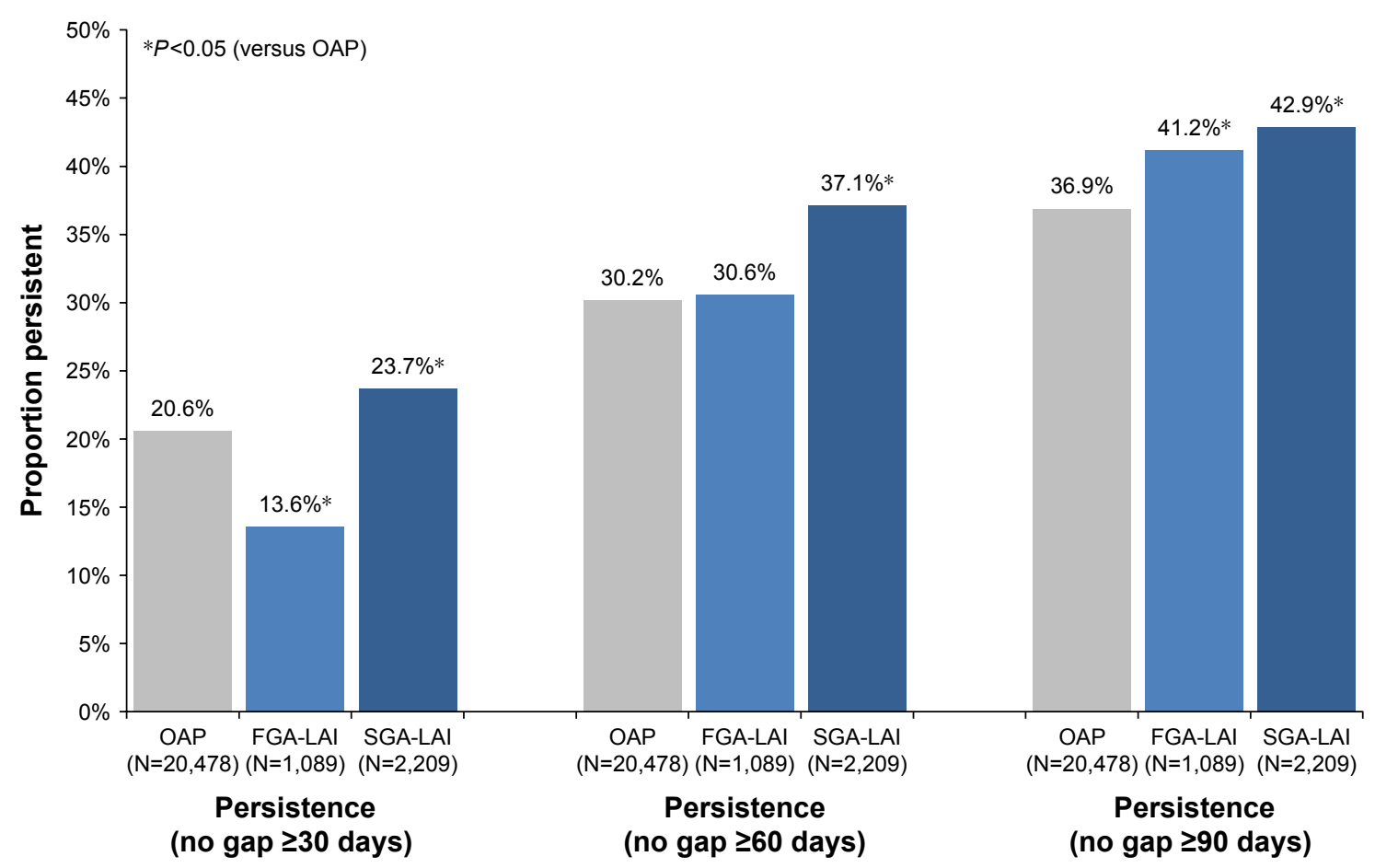

Figure 3 Observed persistence (no gap $\geq 30$ days, $\geq 60$ days, or $\geq 90$ days) on index treatment during the 12 -month follow-up period.

Abbreviations: FGA-LAI, first-generation long-acting injectable therapies; OAP, oral antipsychotic; SGA-LAI, second-generation long-acting injectable therapies.

2010 or later reported that patients initiated on SGA-LAI were more likely to be adherent and persistent to their medication than patients initiated on OAP, while such difference was not as clear for patients initiated on FGA-LAI. Patients initiated on SGA-LAI were also less likely to use another AP medication and less likely to have AP polypharmacy during follow-up compared to patients initiated on OAP, while FGALAI patients were more likely to have AP polypharmacy compared to OAP patients.

The finding that initiating SGA-LAI over OAP was associated with improved adherence and persistence is consistent with the recent retrospective study by Marcus et al who

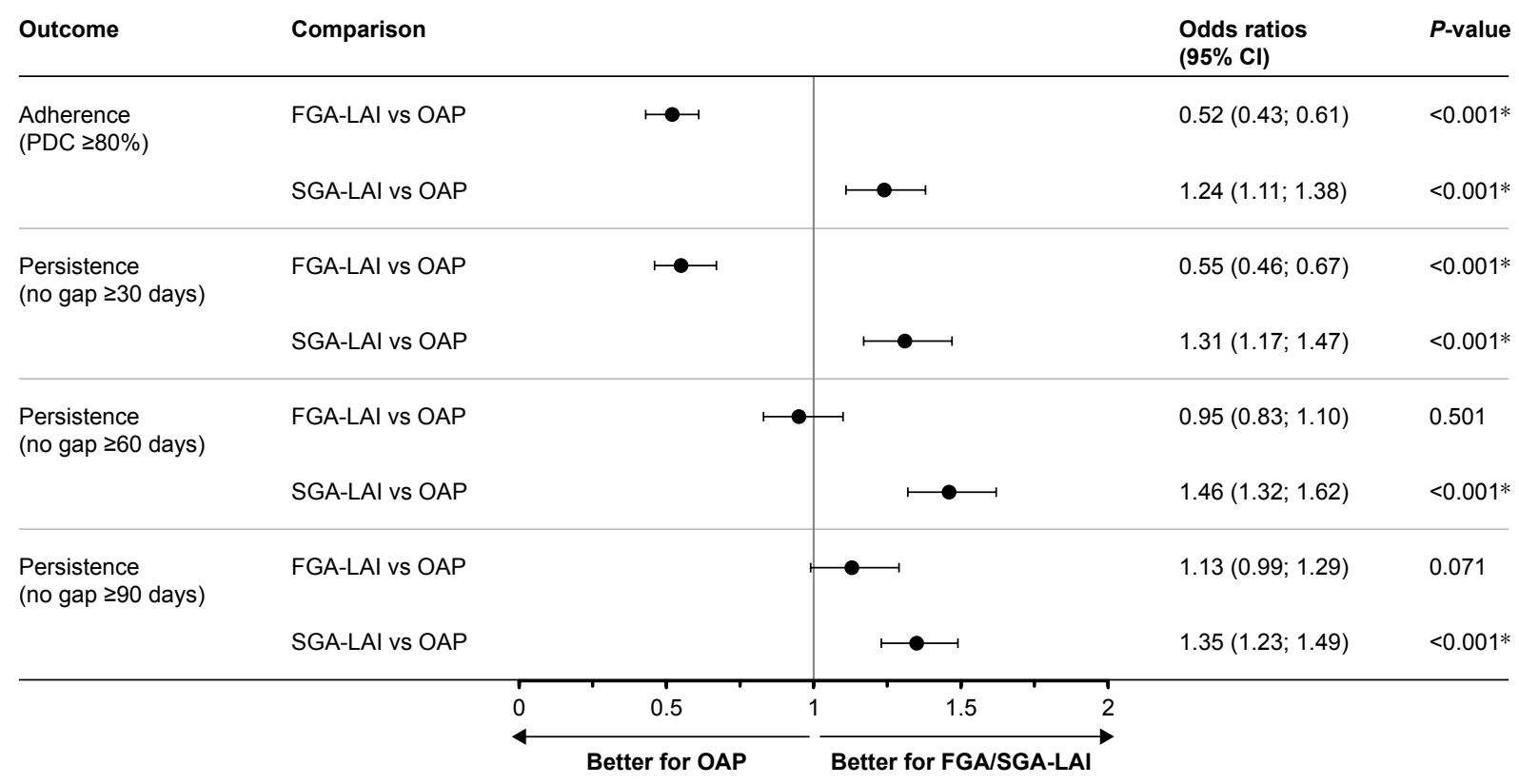

Figure 4 Multivariable comparison of adherence and persistence outcomes during the 12-month follow-up period. Note: *P-value $<0.05$ (versus OAP).

Abbreviations: Cl, confidence interval; FGA-LAI, first-generation long-acting injectable therapies; OAP, oral antipsychotics; PDC, proportion of days covered; SGA-LAI, second-generation long-acting injectable therapies. 
compared AP medication adherence and re-hospitalization in a population of 3,428 OAP versus 340 LAI Medicaid patients with schizophrenia with a history of non-adherence and a recent schizophrenia-related hospitalization. ${ }^{18}$ In that study after adjusting for observable characteristics, the authors identified that SGA-LAI patients were $68 \%$ less likely (OR: $0.32 ; P<0.001$ ) to be non-adherent and were significantly less likely to incur a gap in treatment of more than 60 days (OR: $0.34 ; P<0.001)$ compared to OAP patients. ${ }^{18}$ Generally, the present study found lower rates of adherence (16\%-27\%) compared to the study by Marcus et al $(32 \%-53 \%)$. This is likely explained by the longer observation in the present study (12 vs 6 months); adherence rates at 6 months (data not shown) were more similar to the range reported previously. However, other factors including differences in the underlying patient population may have also contributed. The present study, which included a larger and broader population with schizophrenia and used more recent data with newer SGA-LAI agents, further supports the finding that SGA-LAI is associated with improved adherence and persistence over OAP in real-world clinical settings.

However, the present study did not find evidence to support that FGA-LAI also confers a significant improvement over OAP in terms of these outcomes, which is in contrast to the study by Marcus et al as well as other studies that suggested that FGA-LAI may reduce the risk of treatment discontinuation and may improve adherence as compared to OAP. ${ }^{18-21}$ After accounting for baseline characteristics, FGA-LAI patients in the current study were found to be significantly less adherent to medication than OAP patients, and persistence compared to OAP patients varied depending on the minimum gap of treatment used (the smaller the gap, the less favorable was the outcome for FGA-LAI vs OAP patients). One possible explanation for the discrepancy between the present findings and those presented by Marcus et al may be related to differences in the patient population; Marcus et al limited their sample to patients with a schizophrenia-related hospitalization and recent nonadherence to oral APs, suggesting that their population may have included more severe, non-adherent patients than the present study. In addition, while Marcus et al demonstrated that FGA-LAI was associated with better adherence compared to FGA-OAP, the comparator group of the present study comprised 79\% SGA-OAP. SGA-OAP may have a favorable side effect profile compared to FGA-OAP, potentially explaining why the present findings were less favorable for FGA-LAI compared to those by Marcus et al. One meta-analysis of observational studies has also demonstrated variability in findings of studies comparing FGA-LAI versus OAP, with some studies reporting better, worse, or no difference between treatment groups for outcomes such as relapse and discontinuation, supporting the notion that such analyses are sensitive to the patient population and study design used. ${ }^{22}$

Results from this and previous studies therefore suggest that SGA-LAI may be associated with greater impacts on adherence versus OAP than FGA-LAI, although future investigations are needed in order to make more direct comparisons, particularly for outcomes subsequent to adherence (eg, relapse and re-hospitalization). While both FGA-LAI and SGA-LAI are administered less frequently than OAP, which would be expected to improve treatment adherence, we hypothesize that the side effect profiles of FGA versus SGA, in particular the higher incidence of extrapyramidal symptoms associated with FGA, may partly explain observed differences in adherence and persistence. ${ }^{23}$

These results are of key importance particularly given that poor adherence to medication is an important driver of relapse in schizophrenic patients ${ }^{11}$ and that relapses are associated with a significant increase in health care costs mostly due to institutional visits (ie, hospitalizations and emergency department visits). ${ }^{24,25}$ Reasons for poor adherence to AP medication in patients with schizophrenia have been largely investigated and include, in addition to factors related to the medication itself, lack of illness awareness, direct impacts of symptoms, social isolation, comorbid substance misuse, stigma, and the increasing fragmentation of mental health services in many countries. ${ }^{10}$ LAI can help overcome several of these barriers to adherence by alleviating some of the burden of treatment delivery experienced by the patients and reducing the frequency of administration. They are one rational approach to addressing non-adherence issues. ${ }^{26}$ As a matter of fact, several real-world studies showed the positive impact of LAI, especially SGA-LAI, as compared to OAP, on hospitalization, health care resource use, and costs. ${ }^{13,15,16,27}$

Lastly, the current findings also demonstrate that while patients initiated on SGA-LAI were more adherent and persistent to treatment than those initiated on OAP, they required less use of additional psychiatric medications such as APs, anxiolytics, antidepressants, and mood stabilizers. The observed reduction in polypharmacy associated with SGA-LAI further substantiates this. In addition to reducing medication-associated costs, these findings may also suggest that SGA-LAI patients may experience less of a burden related to concomitant medication use, including fewer side effects and lower risks of drug-drug interactions, which could increase health care costs and resource use and have negative impacts on AP adherence and schizophrenia symptom management. 
The current study was subject to certain limitations that warrant mention. First, the Medicaid data used in this study came from only six states and may not be representative of the United States, other states, or non-Medicaid patients. Second, administrative claims data, including the data used in this study, may be subject to missing data, coding inaccuracies, and residual confounding between treatment cohorts due to unmeasured confounders. Third, claims-based adherence measures such as PDC or persistence do not account for whether the drugs dispensed were actually taken as prescribed. This may overestimate patient adherence, especially for those taking oral AP medication, for whom we assumed that they take their medication correctly (eg, one pill per day), whereas for LAI, the duration of effect for one injection is independent of any further action by the patient. Fourth, results did not account for the pharmacokinetic profiles of different LAI and OAP agents. Specifically, because LAI typically have longer half-lives, patients receiving these therapies may still have active treatment up to several weeks following their last $\operatorname{dos}^{26}$ unlike patients on OAP who typically no longer have significant active treatment after a few days. Therefore, gaps in treatment used to define discontinuation may need to be longer for LAI than for OAP to be clinically meaningful. Of note, these latest two limitations are expected to have a conservative impact on the findings of the study. While health insurance claims data do present such shortcomings, they remain a valuable source of information because they contain a fairly valid and large sample of patients' characteristics and outcomes in a real-world setting. Fifth, this study used an intent-to-treat approach that did not account for treatment switching or discontinuation after the index date. In particular, the impact of LAI on adherence may have been underestimated due to LAI use among OAP patients during follow-up. Lastly, the OAP comparison group in this study comprised patients initiated on both FGA and SGA-OAP, with the majority having initiated on SGA-OAP (78\%). This comparison group was constructed based on observed treatment patterns and is perhaps more representative of patients treated with OAP in the real world than a population matched to each LAI cohort by generation of agents. To assess the impact of comparing each LAI cohort to a mix of FGA and SGA OAP, we performed a sensitivity analysis of adherence and persistence outcomes that only compared each LAI cohort with its respective generation of OAP (ie, FGA-LAI vs FGA-OAP and SGA-LAI vs SGA-OAP) and found similar results.

\section{Conclusion}

Among Medicaid patients with schizophrenia, initiation of SGA-LAI was associated with better adherence and persistence as compared to OAP. Conversely, initiation of FGA-LAI was associated with poor adherence and similar persistence when compared to OAP. Additionally, patients initiated on FGA-LAI were more likely to have AP polypharmacy during follow-up as compared to patients initiated on OAP. The present study adds to the growing body of realworld based literature that shows the benefits of SGA-LAI over OAP, in particular in terms of adherence to treatment and, thereby, treatment efficacy. Further studies are warranted to understand the impact of these AP treatments on clinical and economic outcomes.

\section{Acknowledgment}

Part of the material in this manuscript was presented at the 169th American Psychiatric Association Annual Meeting, May 14-18, 2016, Atlanta, GA, USA.

\section{Author contributions}

All the authors contributed to the conception and design of the current study. Analyses were carried out by DP, MHL, RK, BE, and PL. All the authors were involved in interpreting the findings and drafting the manuscript, and all approved the final version.

\section{Disclosure}

DP, MHL, RLK, BE, and PL are employees of Analysis Group, Inc., a consulting company that has received research grants from Janssen Scientific Affairs, LLC, to conduct this study. KJ and NT are employees of Janssen Scientific Affairs, LLC. The authors report no other conflicts of interest in this work.

\section{References}

1. World Health Organization. WHO. Schizophrenia [Internet]. Available from: http://www.who.int/mental_health/management/schizophrenia/en/. Accessed July 21, 2016.

2. National Institute of Mental Health. Schizophrenia. Available from: http:// www.nimh.nih.gov/health/statistics/prevalence/schizophrenia.shtml. Accessed July 21, 2016.

3. Millier A, Schmidt U, Angermeyer MC, et al. Humanistic burden in schizophrenia: a literature review. J Psychiatr Res. 2014;54:85-93.

4. Carr VJ, Lewin TJ, Neil AL, Halpin SA, Holmes S. Premorbid, psychosocial and clinical predictors of the costs of schizophrenia and other psychoses. Br J Psychiatry. 2004;184:517-525.

5. Ascher-Svanum H, Zhu B, Faries DE, et al. The cost of relapse and the predictors of relapse in the treatment of schizophrenia. BMC Psychiatry. 2010;10:2.

6. Cloutier M, Aigbogun MS, Guerin A, et al. The economic burden of schizophrenia in the United States in 2013. J Clin Psychiatry. 2016;77(6): 764-771. 
7. American Psychiatric Association. Practice Guideline for the Treatment of Patients With Schizophrenia. 2nd ed. Washington, DC: American Psychiatric Association; 2004.

8. Kishimoto T, Agarwal V, Kishi T, Leucht S, Kane JM, Correll CU. Relapse prevention in schizophrenia: a systematic review and metaanalysis of second-generation antipsychotics versus first-generation antipsychotics. Mol Psychiatry. 2013;18(1):53-66.

9. Correll CU, Leucht S, Kane JM. Lower risk for tardive dyskinesia associated with second-generation antipsychotics: a systematic review of 1-year studies. Am J Psychiatry. 2004;161(3):414-425.

10. Haddad PM, Brain C, Scott J. Nonadherence with antipsychotic medication in schizophrenia: challenges and management strategies. Patient Relat Outcome Meas. 2014;5:43-62.

11. Olivares JM, Sermon J, Hemels M, Schreiner A. Definitions and drivers of relapse in patients with schizophrenia: a systematic literature review. Ann Gen Psychiatry. 2013;12(1):32.

12. Kishimoto T, Robenzadeh A, Leucht C, et al. Long-acting injectable vs oral antipsychotics for relapse prevention in schizophrenia: a metaanalysis of randomized trials. Schizophr Bull. 2014;40(1):192-213.

13. Offord S, Wong B, Mirski D, Baker RA, Lin J. Healthcare resource usage of schizophrenia patients initiating long-acting injectable antipsychotics vs oral. J Med Econ. 2013;16(2):231-239.

14. Alphs L, Schooler N, Lauriello J. How study designs influence comparative effectiveness outcomes: the case of oral versus long-acting injectable antipsychotic treatments for schizophrenia. Schizophr Res. 2014;156(2-3):228-232.

15. Kishimoto T, Nitta M, Borenstein M, Kane JM, Correll CU. Long-acting injectable versus oral antipsychotics in schizophrenia: a systematic review and meta-analysis of mirror-image studies. J Clin Psychiatry. 2013;74(10):957-965.

16. Lafeuille MH, Laliberté-Auger F, Lefebvre P, Frois C, Fastenau J, Duh MS. Impact of atypical long-acting injectable versus oral antipsychotics on rehospitalization rates and emergency room visits among relapsed schizophrenia patients: a retrospective database analysis. $B M C$ Psychiatry. 2013;13:221.

17. Lafeuille MH, Grittner AM, Fortier J, et al. Comparison of rehospitalization rates and associated costs among patients with schizophrenia receiving paliperidone palmitate or oral antipsychotics. Am J Health Syst Pharm. 2015;72(5):378-389.
18. Marcus SC, Zummo J, Pettit AR, Stoddard J, Doshi JA. Antipsychotic adherence and rehospitalization in schizophrenia patients receiving oral versus long-acting injectable antipsychotics following hospital discharge. J Manag care Spec Pharm. 2015;21(9):754-768.

19. Brnabic AJ, Kelin K, Ascher-Svanum H, Montgomery W, Kadziola Z, Karagianis J. Medication discontinuation with depot and oral antipsychotics in outpatients with schizophrenia: comparison of matched cohorts from a 12-month observational study. Int J Clin Pract. 2011;65(9):945-953.

20. Shi L, Ascher-Svanum H, Zhu B, Faries D, Montgomery W, Marder SR. Characteristics and use patterns of patients taking first-generation depot antipsychotics or oral antipsychotics for schizophrenia. Psychiatr Serv. 2007;58(4):482-488.

21. Zhu B, Ascher-Svanum H, Shi L, Faries D, Montgomery W, Marder SR. Time to discontinuation of depot and oral first-generation antipsychotics in the usual care of schizophrenia. Psychiatr Serv. 2008; 59(3):315-317.

22. Haddad PM, Taylor M, Niaz OS. First-generation antipsychotic longacting injections v. oral antipsychotics in schizophrenia: systematic review of randomised controlled trials and observational studies. Br J Psychiatry Suppl. 2009;52:S20-S28.

23. Pierre JM. Extrapyramidal symptoms with atypical antipsychotics: incidence, prevention and management. Drug Saf. 2005;28(3):191-208.

24. Lafeuille MH, Gravel J, Lefebvre P, et al. Patterns of relapse and associated cost burden in schizophrenia patients receiving atypical antipsychotics. J Med Econ. 2013;16(11):1290-1299.

25. Sun SX, Liu GG, Christensen DB, Fu AZ. Review and analysis of hospitalization costs associated with antipsychotic nonadherence in the treatment of schizophrenia in the United States. Curr Med Res Opin. 2007;23(10):2305-2312.

26. Brissos S, Veguilla MR, Taylor D, Balanzá-Martinez V. The role of long-acting injectable antipsychotics in schizophrenia: a critical appraisal. Ther Adv Psychopharmacol. 2014;4(5):198-219.

27. Lafeuille MH, Dean J, Carter V, et al. Systematic review of long-acting injectables versus oral atypical antipsychotics on hospitalization in schizophrenia. Curr Med Res Opin. 2014;30(8):1643-1655.
Patient Preference and Adherence

\section{Publish your work in this journal}

Patient Preference and Adherence is an international, peer-reviewed, open access journal that focuses on the growing importance of patient preference and adherence throughout the therapeutic continuum. Patient satisfaction, acceptability, quality of life, compliance, persistence and their role in developing new therapeutic modalities and compounds to optimize

\section{Dovepress}

clinical outcomes for existing disease states are major areas of interest for the journal. This journal has been accepted for indexing on PubMed Central. The manuscript management system is completely online and includes a very quick and fair peer-review system, which is all easy to use. Visit http://www. dovepress.com/testimonials.php to read real quotes from published authors. 